\title{
A Triple-Continuum Pressure-Transient Model for a Naturally Fractured Vuggy Reservoir ${ }^{1}$
}

\author{
Yu-Shu Wu, SPE, Lawrence Berkeley National Laboratory, Christine Ehlig-Economides, SPE, Texas \\ A\&M, Guan Qin, SPE, Texas A\&M, Zhijiang Kang, SINOPEC, Wangming Zhang SINO PEC, Babatunde \\ Ajayi, SPE, Texas A\&M, and Qingfeng Tao, SPE, Texas A\&M
}

\begin{abstract}
We present an analytical approach for pressure transient test analysis in naturally fractured vuggy reservoirs. This analysis approach relies on a triple-continuum concept, using observed geological data from carbonate oil formations in western China, to describe transient flow behavior in fracture-vug-matrix reservoirs. In the conceptual mathematical model, fractured vuggy rock is considered as a triple-continuum medium, consisting of fractures, rock matrix, and vugs (or cavities). Similar to the classical double-porosity model, the fracture continuum is assumed to be responsible for the occurrence of global flow, while vuggy and matrix continua (providing primary storage space) interact locally with each other as well as with globally connected fractures. Furthermore, the triple continua of fractures, matrix, and vugs are assumed to have uniform and homogeneous properties throughout, and intercontinuum flows between them are at pseudosteady state. With these assumptions, we derive analytical solutions in Laplace space for transient flow toward a well in an infinite and finite reservoir with wellbore storage and skin effects. The analytical solutions reveal typical pressure responses in a fracture-vug-matrix reservoir and can be used for estimating vug properties, in addition to fracture and matrix parameters, through properly designed and conducted well tests. As application examples, actual well test data from a fractured-vuggy reservoir in Western China are analyzed using the triple continuum model.
\end{abstract}

\section{Introduction}

Since the 1960s, significant progress has been made towards understanding and modeling of flow processes in fractured rock [Barenblatt et al., 1960; Warren and Root, 1963; Kazemi, 1969; Pruess and Narasimhan, 1985]. However, most of these studies have focused primarily on naturally fractured reservoirs without taking into consideration large cavities. Recently, characterizing vuggy fractured rock has received attention, because a number of fractured vuggy reservoirs have been found worldwide that can significantly contribute to oil and gas reserves and production [Kossack and Curpine, 2001; Rivas-Gomez et al., 2001; Liu et al. 2003; Hidajat et al., 2004; CamachoVelazquez et al., 2005; Kang et al. 2006; Wu et al. 2006].

Among the commonly used mathematical methods for modeling flow through fractured rock, dual-continuum models (i.e., double- and multi-porosity, and dual-permeability) are perhaps the most popular approaches used in reservoir modeling studies. In addition to the traditional double-porosity concept, a number of triple-porosity or triple-continuum models have been proposed [Closemann, 1975; Wu and Ge, 1983; Abdassah, and Ershaghis, 1986; Bai et al. 1993; Wu et al., 2004; Kang et al. 2006; Wu et al. 2006] to describe flow through fractured rock. In particular, Liu et al. [2003] and Camacho-Velazquez et al. [2005] present several new triple-continuum models for single-phase flow in a fracture-matrix system that includes cavities within the rock matrix (as an additional porous portion of the matrix). In general, these models have focused on handling the heterogeneity of the rock matrix or fractures, e.g., subdividing the rock matrix or fractures into two or more subdomains with different properties.

This study develops an analytical model for analyzing transient pressure behavior in naturally fractured vuggy reservoirs and presents our continuing effort in investigating flow processes in naturally fractured vuggy reservoirs [Kang et al. 2006; Wu et al. 2006]. In this study, we focus on single-phase transient flow and fractured vuggy rock, conceptualized also as a triple-or multiple-continuum medium, consisting of (1) highly permeable fractures, (2) lowpermeability rock matrix, and (3) vugs. Similar to the conventional double-porosity model, the fracture continuum is responsible for global flow, while vuggy and matrix continua, providing storage space, are locally connected to each other and interacting with globally connecting fractures. With these assumptions, we derive a mathematical model

1 Funded by National Basic Research Program of China (973 Program), "Studying the Fundamentals of the Carbonate Karst Reservoir Development 2006CB202400.” 
and analytical solutions in the Laplace space for transient flow toward a well in an infinite and finite reservoir. In addition, wellbore storage and skin effects are included. As application examples, actual well test data from a fractured-vuggy reservoir in Western China are analyzed using the triple-continuum model.

\section{Conceptual and Mathematical Models}

As observed in the carbonate formation of the Tahe Oilfield in western China, fractured vuggy reservoirs consist typically of large-scale, well-connected fractures, low-permeable rock matrix, and a large number of cavities or vugs. Those vugs and cavities are irregular in shape and size. Several conceptual models for fractured-vuggy reservoirs are proposed to study flow behavior through such formations in Kang et al. (2006) and Wu et al. (2006). Figure 1 presents a conceptualization for this study, showing that vugs are indirectly connected to fractures through small fractures or microfractures. Similar to the conventional double-porosity concept (Warren and Root, 1963), large fractures are conceptualized to be main pathways for global flow, while vug and matrix continua, locally connected to each other as well as directly or indirectly interacting with globally connecting fractures, generally provide storage space, acting as sinks or sources. Note that vugs and cavities directly connected with fractures are considered part of the fracture continuum. More specifically, we conceptualize the fracture-vug-matrix system as consisting of (1) "large" fractures (or fractures), globally connected on the model scale, (2) various-sized vugs, locally connected to fractures either through "small" fractures (Figure 1) or through rock matrix, and (3) rock matrix, which may contain a number of cavities, locally connected to large fractures and/or vugs.

With these conceptualizations, the Warren-Root model is extended to include one more medium-vugs-in developing the governing equations with the following basic assumptions for obtaining analytical solutions:

1. The reservoir is of uniform thickness with impermeable lower and upper boundaries.

2. The fluid flow from the system into the wellbore is radial, and only the fractures feed the well.

3. All rock properties, such as permeability, initial porosity and compressibility, are constants in each continuum.

4. Fluid flow is isothermal, single-phase, and slightly compressible with constant fluid viscosity.

Furthermore, the quasi-steady-state flow assumption is used for flow between the fracture continuum and the matrix (F-M), and between the vug (V) continuum and the matrix (V-M). Given these assumptions, the flow in a triplecontinuum system can be described as a triple-porosity model (Liu et al. 2003; Wu et al. 2004):

For flow through large fractures:

$$
\frac{\mathrm{k}_{\mathrm{F}}}{\mu} \frac{1}{\mathrm{r}}\left(\mathrm{r} \frac{\partial \mathrm{P}_{\mathrm{F}}}{\partial \mathrm{r}}\right)-\phi_{\mathrm{M}} \mathrm{C}_{\mathrm{M}} \frac{\partial \mathrm{P}_{\mathrm{M}}}{\partial \mathrm{t}}-\phi_{\mathrm{V}} \mathrm{C}_{\mathrm{V}} \frac{\partial \mathrm{P}_{\mathrm{V}}}{\partial \mathrm{t}}=\phi_{\mathrm{F}} \mathrm{C}_{\mathrm{F}} \frac{\partial \mathrm{P}_{\mathrm{F}}}{\partial \mathrm{t}}
$$

For interacting with vugs (or cavities):

$$
\phi_{\mathrm{V}} \mathrm{C}_{\mathrm{V}} \frac{\partial \mathrm{P}_{\mathrm{V}}}{\partial \mathrm{t}}=\frac{\alpha_{\mathrm{FV}} \mathrm{V}_{\mathrm{V}}}{\mu}\left(\mathrm{P}_{\mathrm{F}}-\mathrm{P}_{\mathrm{V}}\right)+\frac{\alpha_{\mathrm{VM}^{\mathrm{k}} \mathrm{M}}}{\mu}\left(\mathrm{P}_{\mathrm{M}}-\mathrm{P}_{\mathrm{V}}\right)
$$

For interacting with the matrix:

$$
\phi_{\mathrm{M}} \mathrm{C}_{\mathrm{M}} \frac{\partial \mathrm{P}_{\mathrm{M}}}{\partial \mathrm{t}}=\frac{\alpha_{\mathrm{FM}} \mathrm{k}_{\mathrm{M}}}{\mu}\left(\mathrm{P}_{\mathrm{F}}-\mathrm{P}_{\mathrm{M}}\right)+\frac{\alpha_{\mathrm{VM}} \mathrm{k}_{\mathrm{M}}}{\mu}\left(\mathrm{P}_{\mathrm{V}}-\mathrm{P}_{\mathrm{M}}\right)
$$

In Equations (1)-(3), subscripts F, M, and V are indexes for fracture, matrix, and vuggy systems, respectively; $\mathrm{P}, \phi$, $\mathrm{C}$, and $\mathrm{k}$ denote pressure, initial porosity, total effective compressibility, and the permeability of each continuum, respectively; $\mu$ is fluid viscosity, and $\alpha_{\mathrm{FV}}, \alpha_{\mathrm{FM}}$ and $\alpha_{\mathrm{VM}}$ are the interporosity flow shape factors. The shape factor for F-M or V-M is defined by Warren and Root (1963):

$$
\alpha_{F M}=\alpha_{V M}=\alpha
$$

For F-V interaction, the shape factor for vugs is defined as

$$
\alpha_{\mathrm{FV}}=\frac{\mathrm{A}_{\mathrm{FV}}}{l_{F V}}
$$

where $\mathrm{A}_{\mathrm{FV}}$ is the total fracture and vug connection area per unit volume of rock $\left(\mathrm{m}^{2} / \mathrm{m}^{3}\right)$ and $l_{\mathrm{FV}}$ is characteristic length, defined as:

$$
l_{\mathrm{FV}}=l_{\mathrm{f}} / 2
$$

where $l_{\mathrm{f}}$ is the average length of small fractures that connect vugs with fractures. 
Initial and Boundary Conditions: The initial pressure $\mathrm{P}_{\mathrm{i}}$ is assumed uniform for the three media throughout the reservoir

$$
\mathrm{P}_{\mathrm{F}}(\mathrm{r}, 0)=\mathrm{P}_{\mathrm{V}}(\mathrm{r}, 0)=\mathrm{P}_{\mathrm{M}}(\mathrm{r}, 0)=\mathrm{P}_{\mathrm{i}}
$$

On outer boundaries, the same constant pressure for a radially infinite system remains:

$$
\mathrm{P}_{\mathrm{F}}(\mathrm{r}=\infty, \mathrm{t})=\mathrm{P}_{\mathrm{i}}
$$

For a radially finite system, the outer boundary (with a radius $=r_{e}$ ) is subject to the following two conditions:

$$
P_{F}\left(r=r_{e}, t\right)=P_{i}
$$

for constant boundary condition; and

$$
\frac{\partial \mathrm{P}\left(\mathrm{r}=\mathrm{r}_{\mathrm{e}}, \mathrm{t}\right)}{\partial \mathrm{r}}=0
$$

for closed outer boundary condition.

The boundary conditions at the wellbore $\left(r=r_{w}\right)$ are decided by a constant volumetric flow rate, $q$, imposed to the well at surface conditions, subject to wellbore storage effects and the effect of an infinitesimal skin region around the wellbore. They can be related by:

$$
\mathrm{P}_{\mathrm{wf}}=\left[\mathrm{P}_{\mathrm{F}}-\mathrm{Sr}_{\mathrm{w}} \frac{\partial \mathrm{P}_{\mathrm{F}}}{\partial \mathrm{r}}\right]_{\mathrm{r}=\mathrm{r}_{\mathrm{W}}}
$$

and

$$
-\mathrm{C} \frac{\partial \mathrm{P}_{\mathrm{wf}}}{\partial \mathrm{t}}+\frac{2 \pi \mathrm{r}_{\mathrm{W}} \mathrm{k}_{\mathrm{F}} \mathrm{h}}{\mu} \frac{\partial \mathrm{P}_{\mathrm{F}}}{\partial \mathrm{r}}\left(\mathrm{r}_{\mathrm{w}}, \mathrm{t}\right)=\mathrm{q}
$$

where $\mathrm{S}$ is a skin factor, dimensionless constant, $\mathrm{C}$ is wellbore storage constant, $\mathrm{P}_{\mathrm{wf}}$ is flowing borehole pressure, $r_{w}$ is wellbore radius, and $h$ the thickness of the uniform, horizontal formation. The wellbore storage constant is defined as:

$$
\mathrm{C}=\mathrm{V}_{\mathrm{w}} \mathrm{c} \mathrm{L}
$$

where $\mathrm{V}_{\mathrm{w}}$ is wellbore volume and $\mathrm{c}_{\mathrm{L}}$ is compressibility of liquid or fluid in wellbore.

Introducing dimensionless pressure, $P_{D}\left(r_{D}, t_{D}\right)$, dimensionless radial distance $r_{D}$, and dimensionless time $t_{D}$, defined as

$$
\begin{gathered}
P_{D}\left(r_{D}, t_{D}\right)=\frac{2 \pi k F_{F} h}{\mu q}\left(P_{i}-P(r, t)\right), \\
r_{D}=\frac{r}{r_{w}},
\end{gathered}
$$

and

$$
{ }^{\mathrm{t}} \mathrm{D}=\frac{\mathrm{t}}{\left[\mu \mathrm{rr}_{\mathrm{W}}^{2}\left(\phi_{\mathrm{F}} \mathrm{C}_{\mathrm{F}}+\phi_{\mathrm{V}} \mathrm{C}_{\mathrm{V}}+\phi_{\mathrm{M}} \mathrm{C}_{\mathrm{M}}\right) / \mathrm{k}_{\mathrm{F}}\right]}
$$

Governing equations (1)-(3), initial conditions (7), and boundary conditions (8)-(12) become

$$
\begin{aligned}
& \omega_{\mathrm{F}} \frac{\partial \mathrm{P}_{\mathrm{DF}}}{\partial \mathrm{t}_{\mathrm{D}}}-\frac{1}{\mathrm{r}_{\mathrm{D}}} \frac{\partial}{\partial \mathrm{r}_{\mathrm{D}}}\left(\mathrm{r}_{\mathrm{D}} \frac{\partial \mathrm{P}_{\mathrm{DF}}}{\partial \mathrm{r}_{\mathrm{D}}}\right)-\lambda_{\mathrm{FV}}\left(\mathrm{P}_{\mathrm{DV}}-\mathrm{P}_{\mathrm{DF}}\right) \\
& -\lambda_{\mathrm{FM}}\left(\mathrm{P}_{\mathrm{DM}}-\mathrm{P}_{\mathrm{DF}}\right)=0
\end{aligned}
$$




$$
\begin{aligned}
& \omega_{\mathrm{V}} \frac{\partial \mathrm{P}_{\mathrm{DV}}}{\partial \mathrm{t}_{\mathrm{D}}}+\lambda_{\mathrm{FV}}\left(\mathrm{P}_{\mathrm{DV}}-\mathrm{P}_{\mathrm{DF}}\right)+\lambda_{\mathrm{VM}}\left(\mathrm{P}_{\mathrm{DV}}-\mathrm{P}_{\mathrm{DM}}\right)=0, \\
& { }^{\omega} \mathrm{M} \frac{\partial \mathrm{P}_{\mathrm{DM}}}{\partial \mathrm{t}_{\mathrm{D}}}+\lambda_{\mathrm{FM}}\left(\mathrm{P}_{\mathrm{DM}}-\mathrm{P}_{\mathrm{DF}}\right)+\lambda_{\mathrm{VM}}\left(\mathrm{P}_{\mathrm{DM}}-\mathrm{P}_{\mathrm{DV}}\right)=0,
\end{aligned}
$$

where $\omega$ 's and $\lambda$ 's are defined in Table 1 .

Initial condition:

$$
\mathrm{P}_{\mathrm{DF}}\left(\mathrm{r}_{\mathrm{D}}, 0\right)=\mathrm{P}_{\mathrm{DV}}\left(\mathrm{r}_{\mathrm{D}}, 0\right)=\mathrm{P}_{\mathrm{DM}}\left(\mathrm{r}_{\mathrm{D}}, 0\right)=0,
$$

Outer boundary conditions:

$$
\begin{gathered}
\mathrm{P}_{\mathrm{DF}}\left(\infty, \mathrm{t}_{\mathrm{D}}\right)=0, \\
\mathrm{P}_{\mathrm{DF}}\left(\mathrm{r}_{\mathrm{D}}=\mathrm{r}_{\mathrm{e}} / \mathrm{r}_{\mathrm{w}}=\mathrm{r}_{\mathrm{eD}}, \mathrm{t}_{\mathrm{D}}\right)=0, \\
\left.\frac{\partial \mathrm{P}_{\mathrm{DF}}}{\partial \mathrm{r}}\right|_{\mathrm{r}_{\mathrm{D}}=\mathrm{r}_{\mathrm{eD}}}=0
\end{gathered}
$$

and inner boundary conditions:

$$
\begin{gathered}
\mathrm{P}_{\mathrm{Dwf}}=\left[\mathrm{P}_{\mathrm{DF}}-\mathrm{S} \frac{\partial \mathrm{P}_{\mathrm{DF}}}{\partial \mathrm{r}_{\mathrm{D}}}\right]_{\mathrm{r}_{\mathrm{D}}=1} \\
\mathrm{C}_{\mathrm{D}} \frac{\partial \mathrm{P}_{\mathrm{Dwf}}}{\partial \mathrm{t}_{\mathrm{D}}}-\left(\frac{\partial \mathrm{P}_{\mathrm{DF}}}{\partial \mathrm{r}_{\mathrm{D}}}\right)_{\mathrm{r}_{\mathrm{D}}=1}=1
\end{gathered}
$$

where

$$
\mathrm{P}_{\text {Dwf }}=\frac{2 \pi \mathrm{k}_{\mathrm{F}} \mathrm{h}}{\mu \mathrm{q}}\left(\mathrm{P}_{\mathrm{i}}-\mathrm{P}_{\mathrm{wf}}\right),
$$

and dimensionless wellbore storage coefficient,

$$
\mathrm{C}_{\mathrm{D}}=\frac{\mathrm{C}}{2 \pi\left(\phi_{\mathrm{F}} \mathrm{c}_{\mathrm{F}}+\phi_{\mathrm{F}^{\mathrm{c}} \mathrm{F}}+\phi_{\mathrm{F}} \mathrm{c}_{\mathrm{F}}\right) \mathrm{hr}_{\mathrm{w}}^{2}},
$$

Laplace Space Solutions: Applying the Laplace transformation to Equations (17) through (25) yields:

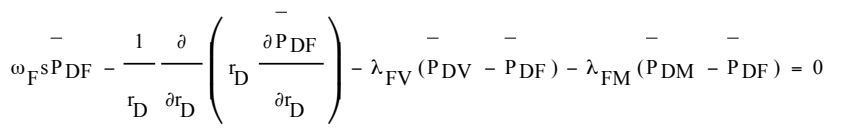

$$
\begin{aligned}
& { }^{\omega}{ }_{\mathrm{V}}{ }^{\overline{\mathrm{P}}} \mathrm{DV}+\lambda_{\mathrm{FV}}\left(\overline{\mathrm{P}}_{\mathrm{DV}}-\overline{\mathrm{P}}_{\mathrm{DF}}\right)+\lambda_{\mathrm{VM}}\left(\overline{\mathrm{P}}_{\mathrm{DV}}-\overline{\mathrm{P}}_{\mathrm{DM}}\right)=0 \\
& \omega_{\mathrm{M}} \mathrm{M}^{\mathrm{sP} \mathrm{DM}}+\lambda_{\mathrm{FM}}\left({ }^{\mathrm{P}} \mathrm{DM}-\overline{\mathrm{P}} \mathrm{DF}\right)+\lambda_{\mathrm{VM}}(\stackrel{-}{\mathrm{P} D M}-\overline{\mathrm{PDV}})=0 \\
& \overline{\mathrm{PDF}}\left(\mathrm{r}_{\mathrm{D}}=\infty, \mathrm{s}\right)=0
\end{aligned}
$$

and 


$$
\begin{aligned}
& \operatorname{PDF}\left(\mathrm{r}_{\mathrm{D}}=\mathrm{r}_{\mathrm{eD}}, \mathrm{s}\right)=0 \\
& \left.\frac{-\overline{\mathrm{PDF}}}{\mathrm{dr}}\right|_{\mathrm{r}_{\mathrm{D}}=\mathrm{r}_{\mathrm{eD}}}=0
\end{aligned}
$$

and inner boundary conditions:

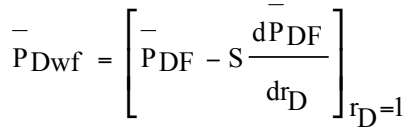

$$
\begin{aligned}
& \left.\mathrm{C}_{\mathrm{D}^{\mathrm{sP}} \stackrel{-}{-}-\left(\frac{-}{\mathrm{dPDF}}\right.}^{\mathrm{dr}_{\mathrm{D}}}\right)_{\mathrm{r}_{\mathrm{D}}=1}=\frac{1}{\mathrm{~s}}
\end{aligned}
$$

where $\overline{\mathrm{P}}_{\mathrm{DF}}, \overline{\mathrm{P}}_{\mathrm{DV}}, \overline{\mathrm{P}}_{\mathrm{DM}}$, and $\overline{\mathrm{P}}_{\mathrm{Dwf}}$ are Laplace transformed functions of $\mathrm{P}_{\mathrm{DF}}, \mathrm{P}_{\mathrm{DV}}, \mathrm{P}_{\mathrm{DM}}$, and $\mathrm{P}_{\mathrm{Dwf}}$ in the Laplace domain, and $\mathrm{S}$ is the Laplace transformation variable.

Substituting the matrix and cavity equations of (29) and (30) into the fracture equation (28), we have:

$$
\frac{1}{{ }_{\mathrm{D}}} \frac{\partial}{\partial \mathrm{r}_{\mathrm{D}}}\left({ }^{\mathrm{r}} \frac{\partial \mathrm{P} \mathrm{DF}}{\partial \mathrm{r}_{\mathrm{D}}}\right)-\mathrm{sf}(\mathrm{s}) \overline{\mathrm{P}} \mathrm{DF}=0
$$

where

$$
\mathrm{f}(\mathrm{s})=\omega_{\mathrm{F}}+\frac{\left(\lambda_{\mathrm{FV}}+\lambda_{\mathrm{FM}}\right)+\frac{1-{ }^{\omega} \mathrm{F}}{{ }^{\omega}{ }_{\mathrm{V}}{ }_{\mathrm{M}}}\left[\lambda_{\mathrm{FV}} \lambda_{\mathrm{FM}}+\left(\lambda_{\mathrm{FV}}+\lambda_{\mathrm{FM}}\right) \lambda_{\mathrm{VM}}\right]}{\mathrm{s}^{2}+\left[\frac{\lambda_{\mathrm{FV}}}{{ }^{\omega} \mathrm{V}}+\frac{\lambda_{\mathrm{FM}}}{{ }^{\omega} \mathrm{M}}+\left(\frac{1}{{ }^{\omega} \mathrm{V}}+\frac{1}{{ }^{\omega}{ }_{\mathrm{M}}}\right) \lambda_{\mathrm{VM}}\right] \mathrm{s}+\frac{\lambda_{\mathrm{FV}} \lambda_{\mathrm{FM}}+\left(\lambda_{\mathrm{FV}}+\lambda_{\mathrm{FM}}\right) \lambda_{\mathrm{VM}}}{{ }^{\omega} \mathrm{V}^{\omega} \mathrm{M}}}
$$

The general solution to Equation (36) is

$$
-\overline{P D F}=C_{0} K_{0}\left(\sqrt{s f(s)} r_{D}\right)+D_{0} I_{0}\left(\sqrt{s f(s)} r_{D}\right)
$$

where $\mathrm{K}_{0}$ and $\mathrm{I}_{0}$ are the modified Bessel functions of the second and first kinds of zero order, respectively.

Infinite Reservoir: The solution subject to boundary conditions Equations (31), (34) and (35) of an infinite reservoir is:

$$
\begin{aligned}
& \bar{P}_{D F}=\frac{K_{0}(\sqrt{s f(s) r})}{s\left\{\sqrt{s f(s)} K_{1}(\sqrt{s f(s)})+C_{D}\left[K_{0}(\sqrt{s f(s)})+s \sqrt{s f(s)} K_{1}(\sqrt{s f(s)})\right]\right.} \\
& \left.\bar{P}_{\text {Dwf }}=\frac{\mathrm{K}_{0}(\sqrt{\mathrm{sf}(\mathrm{s})})+\mathrm{s} \sqrt{\mathrm{sf}(\mathrm{s}) \mathrm{K}_{1}}(\sqrt{\mathrm{sf}(\mathrm{s})})}{\mathrm{s}\left\{\sqrt{\mathrm{sf(s)} \mathrm{K}_{1}}(\sqrt{\mathrm{sf}(\mathrm{s})})+\mathrm{C}_{\mathrm{D}} \mathrm{s}\left[\mathrm{K}_{0}(\sqrt{\mathrm{sf}(\mathrm{s})})+\mathrm{s} \sqrt{\mathrm{sf}(\mathrm{s}) \mathrm{K}_{1}}(\sqrt{\mathrm{sf}(\mathrm{s})})\right.\right.}\right\}
\end{aligned}
$$

Finite Reservoir with Closed Outer Boundary: The solution subject to boundary conditions Equations (32), (34) and (35) of an infinite reservoir is:

$$
{ }_{P D F}=\frac{\left\{\left\{_{0}\left(\sqrt{s f(s)} r_{D}\right) k_{1}\left(\sqrt{s f(s)} r_{e D}\right)+I_{1}\left(\sqrt{s f(s)} r_{e D}\right) k_{0}\left(\sqrt{s f(s) r_{D}}\right)\right\}\right.}{C_{D^{s}}{ }^{2}\{Y-s \sqrt{s f(s) X}\}-s \sqrt{s f(s) X}}
$$




$$
\begin{aligned}
& \bar{P}_{\text {Dwf }}=\frac{\left\{{ }_{0}(\sqrt{s f(s)}) k_{1}\left(\sqrt{s f(s)} r_{e D}\right)+I_{1}\left(\sqrt{s f(s)} r_{e D}\right) k_{0}(\sqrt{s f(s)})\right\}}{C_{D^{s}}{ }^{2}\{Y-s \sqrt{s f(s)} X\}-s \sqrt{s f(s)} X} \\
& -\frac{s \sqrt{s f(s)}\left\{(\sqrt{s f(s)}) k_{1}\left(\sqrt{s f(s)} r_{e D}\right)+I_{1}\left(\sqrt{s f(s)} r_{e D}\right) k_{1}(\sqrt{s f(s)})\right\}}{C_{D^{s}}{ }^{2}\{Y-s \sqrt{s f(s)} X\}-s \sqrt{s f(s) X}}
\end{aligned}
$$

where,

$$
\begin{aligned}
& X=I_{1}(\sqrt{s f(s)}) K_{1}\left(\sqrt{s f(s)} r_{e D}\right)-I_{1}\left(\sqrt{s f(s)} r_{e D}\right) k_{1}(\sqrt{s f(s)}) \\
& Y=I_{0}(\sqrt{s f(s)}) K_{1}\left(\sqrt{s f(s)} r_{e D}\right)+I_{1}\left(\sqrt{s f(s)} r_{e D}\right) k_{0}(\sqrt{s f(s)})
\end{aligned}
$$

Finite Reservoir with Constant-Pressure Outer Boundary: The solution subject to constant-pressure outer boundary conditions without wellbore storage and skin effects rr is

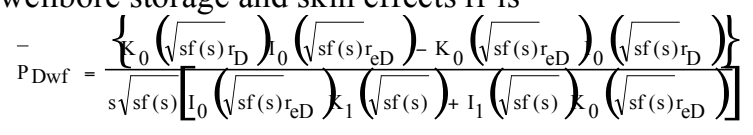

According to Warren and Root (1963), flow through a double-porosity medium can be sufficiently characterized by two parameters, storage parameter $\omega$ and interporosity parameter $\lambda$. The solution in Equation (40) indicates that the flow in the triple-continuum reservoir is characterized by five dimensionless parameters: $2 \omega$ 's and $3 \lambda$ 's (Table 1). Note that only two of the three $\omega$ 's are independent.

\section{Model Behavior}

The analytic solution of Equation (40) can be used to investigate transient flow behavior in fractured vuggy reservoirs. Figure 2 shows basic behavior as well as how wellbore storage affects pressure responses. As discussed in $\mathrm{Wu}$ et al. (2004), flow behavior in a triple-continuum reservoir may exhibit three straight, parallel lines in semi$\log$ space (Figure 2). However, depending on the magnitude of the wellbore storage effect, one or both of the semi$\log$ lines may be masked or distorted.

The characteristic behavior is also observed in the log-log presentation of the logarithmic pressure derivative, with the presence of 2 interporosity flow "valleys". Interporosity flow from the vuggy continuum through the fracture system into the wellbore is observed first, because of its higher interporosity transmissivity parameter $\lambda$ 's, and is identified by the initial valley in the early time region. Then, if the contrast in the interporosity flow parameter is large enough, the second valley, corresponding to fluid transfer from the matrix continuum to the fracture system, is observed later. It can be observed from Figure 3 that it is possible, in some cases, for the wellbore storage to completely mask the presence of the first valley produced by flow from the vuggy continuum. In such cases, the pressure transient test may be wrongfully interpreted as a double-porosity reservoir, thereby effectively ignoring the contribution of the vugs. It is even possible for wellbore storage to mask both valleys, giving the false impression of a homogeneous reservoir.

The fraction of oil reserves in a vuggy fractured reservoir contributed by vuggy porosity may be small when compared with the matrix contribution. However, the computation of the additional porosity due to the vug continuum will cause a noticeable increase in the estimated reserves. In the example used in Figure 3 (parameters given in Table 2), the addition of the vuggy continuum contribution masked by the wellbore storage increases the effective porosity of the reservoir by $13 \%$ and would consequently increase the reserves estimate by as much as $10 \%$ or more, depending on the method of reserves estimation.

\section{Field Examples}

Pressure transient data from two oil-production wells (T313 and TK609) in the Tahe Reservoir, a carbonate, naturally fractured reservoir in western China, exhibit apparent triple-porosity behavior. For these two wells, drilling records, core samples, and geophysical data all indicated that the associated geologic formations are typical of fractured, vuggy rock. The two wells were completed in 2000 and 2001, respectively, with completion depths more than $5,000 \mathrm{~m}$. Buildup tests were performed for both wells and are used here to demonstrate the application of the proposed pressure-transient-analysis approach.

Figures 4 and 5 present matches for the buildup data from the two wells using the triple continuum model. As shown in Figures 4 and 5, model results reasonably match both measured pressure and its derivative data from the 
wells. In both of these examples, the buildup duration was not sufficiently long to observe all of the second characteristic valley, representing interporosity flow from the matrix to natural fractures. As a result, both of these tests can also be matched with a dual-porosity model by matching the falling derivative in late time as a constantpressure boundary. Figure 6 illustrates that the data for Well T313 can be matched in this way. In fact, the dualporosity model provides a better match for these data.

The matches for T313 and TK609 testing data provide the parameters listed in Table 3. An increase in effective porosity from vugs is also indicated in the table, and the increase in reserves after accounting for vugs is quite substantial for T313. The matrix porosity used for the analysis of TK 609 is very small and seems inconsistent with the permeability value determined from the match. All three matches for the two wells show very negative skins that cannot be explained by usual mechanisms. More work is needed to explain the skin results. The dual-porosity match for T313 is achieved with a constant-pressure boundary $6,070 \mathrm{ft}$ from the well. The triple-porosity model does not show any reservoir limit up to the end of the buildup.

\section{Summary and Concluding Remarks}

A physically based conceptual and mathematical model is presented for analyzing flow through fractured vuggy rock using a multiple-continuum-medium concept. The proposed multiple-continuum model is a natural extension of the classic double-porosity model, with the fracture continuum responsible for conducting global flow, while vuggy and matrix continua, locally connected and interacting with globally connecting fractures, provide storage space for fluids. In particular, analytical solutions including wellbore storage and skin effects are obtained in Laplace space for pressure-transient analyses in such reservoirs.

As an application example, the proposed well testing approach is demonstrated on actual buildup data for two wells from a naturally fractured vuggy oil reservoir, with reasonable results obtained.

\section{Acknowledgments}

This work was supported by National Basic Research Program of China (973 Program), "Studying the Fundamentals of the Carbonate Karst Reservoir Development 2006CB202400." It was also supported in part by the Research Inst. of Petroleum Exploration and Development of Sinopec Corp, by Texas A\&M University, and by the Lawrence Berkeley National Laboratory through the U.S. Department of Energy under Contract No. DE-AC02$05 \mathrm{CH} 11231$.

\section{References}

Abdassah, D. and Ershaghis I., Triple-porosity system for representing naturally fractured reservoirs, SPE Form. Eval., 1, 113-127, 1986.

Bai, M., Elsworth, D., and Roegiers, J. C., Multiporosity/multipermeability approach to the simulation of naturally fractured reservoirs, Water Resour. Res. 29, 1621-1633, 1993.

Barenblatt, G.I., Zheltov, I.P., and Kochina, I.N., Basic concepts in the theory of seepage of homogeneous liquids in fissured rocks, PMM, Sov. Appl. Math. Mech., 24(5), 852-864, 1960.

Camacho-Velazquez, R., M. Vasquez-Cruz, R. Castrejon-Aivar, and V. Arana-Ortiz, Pressure transient and declinecurve behavior in naturally fractured vuggy carbonate reservoirs, SPE Reservoir Evaluation \& Engineering, 95-111, April, 2005.

Closemann, P.J., The aquifer model for fissured fractured reservoir, Soc. Pet. Eng. J., 385-398, 1975.

Hidajat, I, K. K. Mohanty, M. Flaum, and G. Hirasaki, Study of vuggy carbonates using NMR and X-Ray CT scanning, SPE Reservoir Evaluation \& Engineering, 365-377, October, 2004.

Kang, Zhijiang, Yu-Shu Wu, Jianglong Li, Yongchao Wu, Jie Zhang, and Guangfu Wang, Modeling multiphase flow in naturally fractured vuggy petroleum reservoirs, PSE-1-2356, Presented at the 2006 SPE Annual Technical Cobference and Exhibition, San Antonio, Texas, 24-27 Sempember, 2006.

Kazemi, H., Pressure Transient Analysis of Naturally Fractured Reservoirs with Uniform Fracture Distribution. SPEJ, 451-462. Trans., AIME, 246, 1969. 
Kossack and Curpine, A methodology for simulation of vuggy and fractured reservoirs, SPE-66366, Presented at the SPE Reservoir Simulation Symposium, Houston, Texas, 11-14, February, 2001.

Liu, J.C. G.S. Bodvarsson, and Y.S. Wu, Analysis of pressure behavior in fractured lithophysal reservoirs, Journal of Contaminant Hydrology, 62-62, 189-211, 2003

Pruess, K. and Narasimhan, T.N., A practical method for modeling fluid and heat flow in fractured porous media, Soc. Pet. Eng. J., 25, 14-26, 1985.

Rivas-Gomez et al., Numerical simulation of oil displacement by water in a vuggy fractured porous medium, SPE66386, Presented at the SPE Reservoir Simulation Symposium, Houston, Texas, 11-14, February, 2001.

Warren, J.E. and Root, P.J., The behavior of naturally fractured reservoirs, Soc. Pet. Eng. J., 245-255, Trans., AIME, 228, 1963.

Wu, Y. S., G. Qin, Richard E. Ewing, Yalchin Efendiev, Zhijiang Kang and Yulin Ren, "A Multiple-Continuum Approach for Modeling Multiphase Flow in Naturally Fractured Vuggy Petroleum Reservoirs," SPE104173, Presented at the 2006 SPE International Oil \& Gas Conference and Exhibition, Beijing, China, 5-7 December, 2006.

Wu, Y. S., H. H. Liu, And G. S. Bodvarsson, A triple-continuum approach for modeling flow and transport processes in fractured rock, Journal Of Contaminant Hydrology, 73, 145-179, 2004.

Wu, Y. S. and J. L. Ge, The transient flow in naturally fractured reservoirs with three-porosity systems, Acta, Mechanica Sinica, Theoretical and Applied Mechanics, Beijing, China, 15(1), 81-85, 1983. 
Table 1. Dimensionless parameters and variables used in the analytical solutions of flow through a triplecontinuum reservoir

\begin{tabular}{|c|c|}
\hline Parameter & Definition \\
\hline Dimensionless time & $\mathrm{t}_{\mathrm{D}}=\frac{\mathrm{k}_{\mathrm{F}} \mathrm{t}}{\mu \mathrm{r}_{\mathrm{w}}^{2}\left(\phi_{\mathrm{M}} \mathrm{C}_{\mathrm{M}}+\phi_{\mathrm{V}} \mathrm{C}_{\mathrm{V}}+\phi_{\mathrm{F}} \mathrm{C}_{\mathrm{F}}\right)}$ \\
\hline Dimensionless radius & $r_{D}=\frac{r}{r_{w}}$ \\
\hline Dimensionless pressure & $P_{D}=\frac{\left.P_{i}-P_{F}(r, t)\right)}{\frac{q \mu}{2 \pi k_{F} h}}$ \\
\hline F-M interporosity parameter & $\ddot{\mathrm{e}}_{\mathrm{FM}}=\frac{\alpha_{\mathrm{FM}} \mathrm{r}_{\mathrm{w}}^{2} \mathrm{k}_{\mathrm{M}}}{\mathrm{k}_{\mathrm{F}}}$ \\
\hline F-V interporosity parameter & $\ddot{\mathrm{e}}_{\mathrm{FV}}=\frac{\alpha_{\mathrm{FV}} \mathrm{r}_{\mathrm{w}}^{2} \mathrm{k}_{\mathrm{V}}}{\mathrm{k}_{\mathrm{F}}}$ \\
\hline V-M interporosity parameter & $\ddot{\mathrm{e}}_{\mathrm{VM}}=\frac{\alpha_{\mathrm{VM}} \mathrm{r}_{\mathrm{w}}^{2} \mathrm{k}_{\mathrm{M}}}{\mathrm{k}_{\mathrm{F}}}$ \\
\hline F Storativity & $\grave{\mathrm{u}}_{\mathrm{F}}=\frac{\phi_{\mathrm{F}} \mathrm{C}_{\mathrm{F}}}{\phi_{\mathrm{M}} \mathrm{C}_{\mathrm{M}}+\phi_{\mathrm{V}} \mathrm{CV}+\phi_{\mathrm{F}} \mathrm{C}_{\mathrm{F}}}$ \\
\hline V Storativity & $\grave{\mathrm{u}}_{\mathrm{V}}=\frac{\phi_{\mathrm{V}} \mathrm{C}_{\mathrm{V}}}{\phi_{\mathrm{M}} \mathrm{C}_{\mathrm{M}}+\phi_{\mathrm{V}} \mathrm{C}_{\mathrm{V}}+\phi_{\mathrm{F}} \mathrm{C}_{\mathrm{F}}}$ \\
\hline M Storativity & $\grave{\mathrm{u}}_{\mathrm{M}}=\frac{\phi_{\mathrm{M}} \mathrm{C}_{\mathrm{M}}}{\phi_{\mathrm{M}} \mathrm{C}_{\mathrm{M}}+\phi_{\mathrm{V}} \mathrm{C}_{\mathrm{V}}+\phi_{\mathrm{F}} \mathrm{C}_{\mathrm{F}}}$ \\
\hline \multicolumn{2}{|c|}{ Variables Based on Parameters Listed Above } \\
\hline $\begin{array}{r}\mathrm{A}_{1}=\mathrm{A}_{0}+\frac{\lambda_{\mathrm{FM}}}{2} \\
\mathrm{~A}_{2}=\mathrm{A}_{0}+\frac{\lambda_{\mathrm{FM}}}{2 \mathrm{c}}\end{array}$ & $\begin{array}{l}\left.\left.+\frac{\lambda_{\mathrm{FM}}+\lambda_{\mathrm{FV}}}{2 \omega_{\mathrm{F}}}\right)^{2}-\frac{\mathrm{B}_{0}}{\omega_{\mathrm{F}}}\right]^{1 / 2} \\
\left.\left.+\frac{\lambda_{\mathrm{FM}}+\lambda_{\mathrm{FV}}}{2 \omega_{\mathrm{F}}}\right)^{2}-\frac{\mathrm{B}_{0}}{\omega_{\mathrm{F}}}\right]^{1 / 2}\end{array}$ \\
\hline \multicolumn{2}{|c|}{$\begin{array}{l}\mathrm{B}_{1}=\mathrm{A}_{0}+\left(\mathrm{A}_{0}^{2}-\mathrm{B}_{0}\right)^{/ 2} \\
\mathrm{~B}_{2}=\mathrm{A}_{0}-\left(\mathrm{A}_{0}^{2}-\mathrm{B}_{0}\right)^{/ 2}\end{array}$} \\
\hline $\begin{array}{r}\mathrm{A}_{0}=\frac{1}{2} \\
\mathrm{~B}_{0}=\end{array}$ & $\frac{\left(\frac{1}{\omega_{\mathrm{M}}}+\frac{1}{\omega_{\mathrm{V}}}\right) \lambda_{\mathrm{VM}}}{\left.{ }_{\mathrm{FM}}+\lambda_{\mathrm{FV}}\right) \lambda_{\mathrm{VM}}}$ \\
\hline
\end{tabular}


Table 2: Triple continuum model parameters used to generate the WBS example

\begin{tabular}{|l|l|l|}
\hline Well & Example 1 & \\
\hline $\mathrm{C}$ & varying & $\mathrm{bbl} / \mathrm{psi}$ \\
\hline $\mathrm{Pi}$ & 9000 & $\mathrm{psia}$ \\
\hline $\mathrm{k} . \mathrm{h}$ & 296 & $\mathrm{md} . \mathrm{ft}$ \\
\hline $\mathrm{k}$ & 9.86 & $\mathrm{md}$ \\
\hline Skin & 2 & -- \\
\hline F-M interporosity parameter & & \\
& $5.0 \mathrm{E}-09$ & \\
\hline F-V interporosity parameter & $3.0 \mathrm{E}-07$ & \\
\hline F Storativity & $1.00 \mathrm{E}-02$ & \\
\hline V Storativity & 0.1188 & \\
\hline M Storativity & 0.8712 & \\
\hline
\end{tabular}

Table 3: Triple-continuum and double-continuum model parameters used to match field-case examples

\begin{tabular}{|c|c|c|c|c|}
\hline Well & $\begin{array}{l}\text { T313 Double- } \\
\text { Porosity Match }\end{array}$ & T313 & TK609 & \\
\hline $\mathrm{C}$ & 0.0186 & 0.0251 & 0.0973 & $\mathrm{bbl} / \mathrm{psi}$ \\
\hline $\mathrm{Pi}$ & 8246.32 & 8225.55 & 7627.85 & psia \\
\hline k.h & 14200 & 14200 & 22100 & md.ft \\
\hline $\mathrm{k}$ & 193 & 193 & 337 & md \\
\hline Skin & -6.31 & -7.36018 & -10 & -- \\
\hline F-M interporosity parameter & $1.44 \mathrm{E}-06$ & $1.5 \mathrm{E}-09$ & 4.7E-12 & \\
\hline F-V interporosity parameter & -- & $2.0 \mathrm{E}-07$ & 1.0E-09 & \\
\hline F Storativity & 8.84E-04 & $4.31 \mathrm{E}-05$ & $1.69 \mathrm{E}-10$ & \\
\hline V Storativity & -- & 0.1437 & 0.0033 & \\
\hline M Storativity & 0.999 & 0.8563 & 0.9967 & \\
\hline Matrix porosity & 0.15 & 0.15 & 0.02 & \\
\hline Vuggy Porosity & 0 & 0.025172 & $6.65 \mathrm{E}-05$ & \\
\hline$\%$ increase in effective porosity & $0 \%$ & $16.8 \%$ & $0.33 \%$ & \\
\hline Effective Porosity & 0.15 & 0.175 & 0.02 & \\
\hline
\end{tabular}




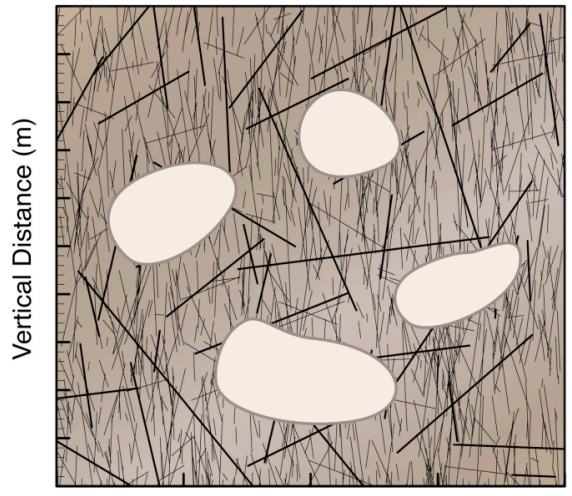

Horizontal Distance (m)

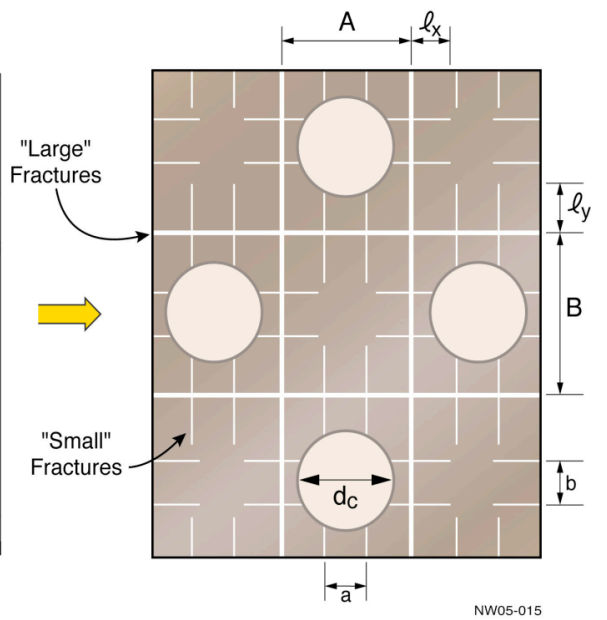

Figure 1. Conceptualization of vuggy fractured rock as a triple-continuum system, with vugs indirectly connected to fractures through small fractures

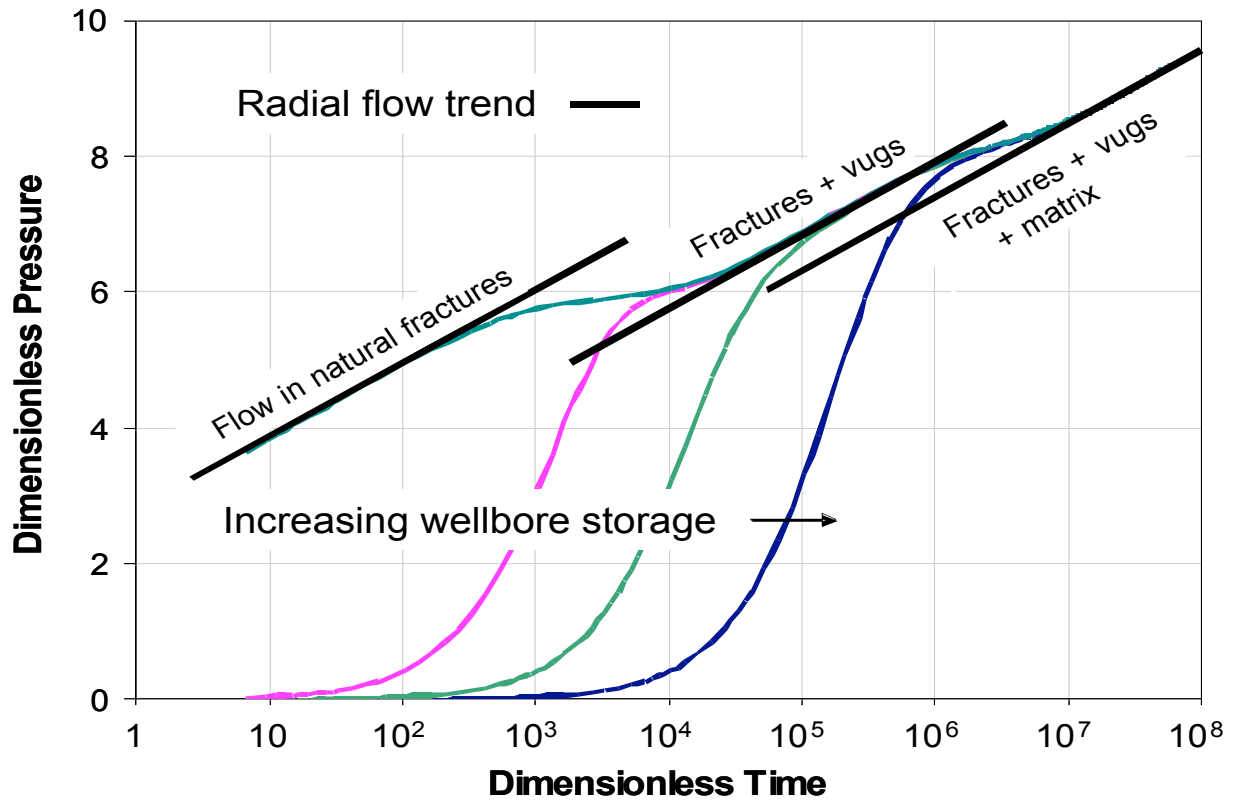

Figure 2. Pressure-transient behavior of flow through a fractured vuggy media, showing triple-continuum flow behavior and the effects of wellbore storage 


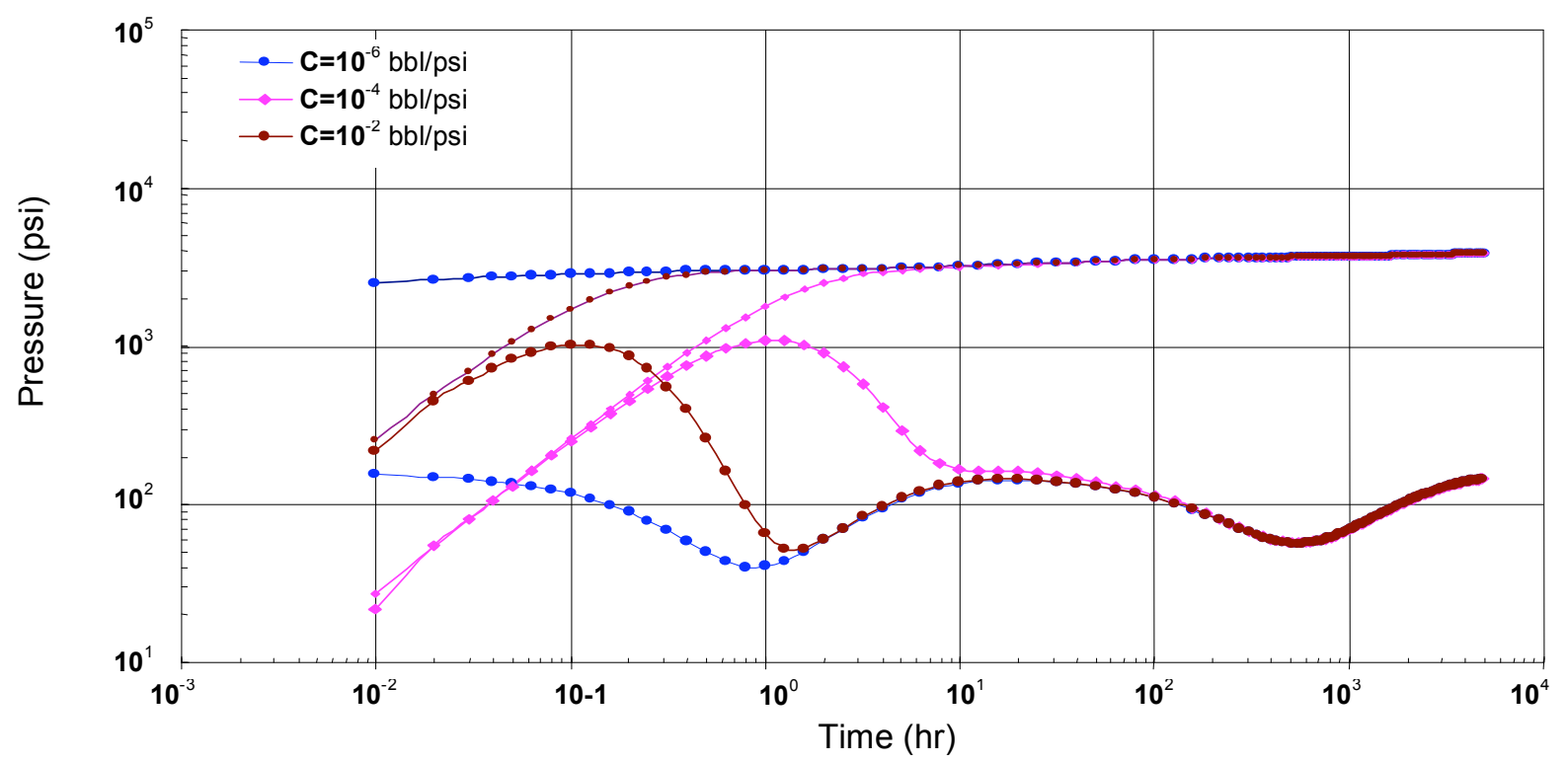

Figure 3. Effects of wellbore storage on pressure-transient behavior in fractured vuggy media (the lower three curves for pressure derivatives)

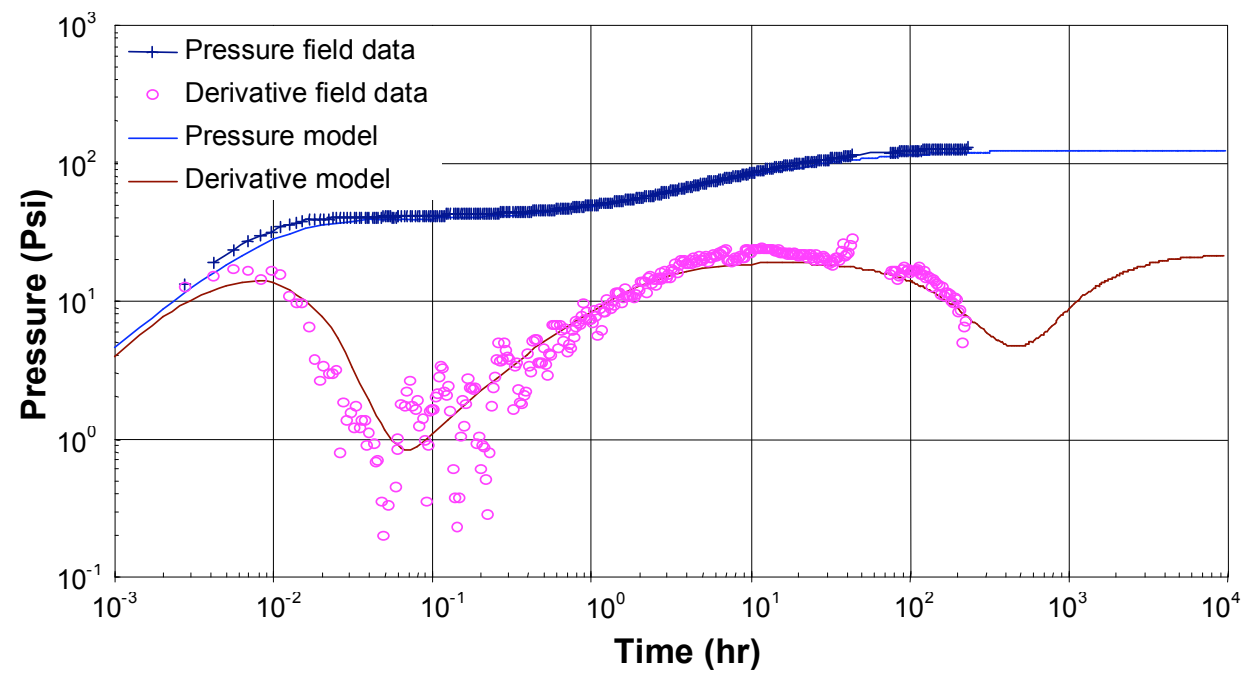

Figure 4. Comparison between measured pressure drawdown and its derivative data, and model predictions for Well T313 


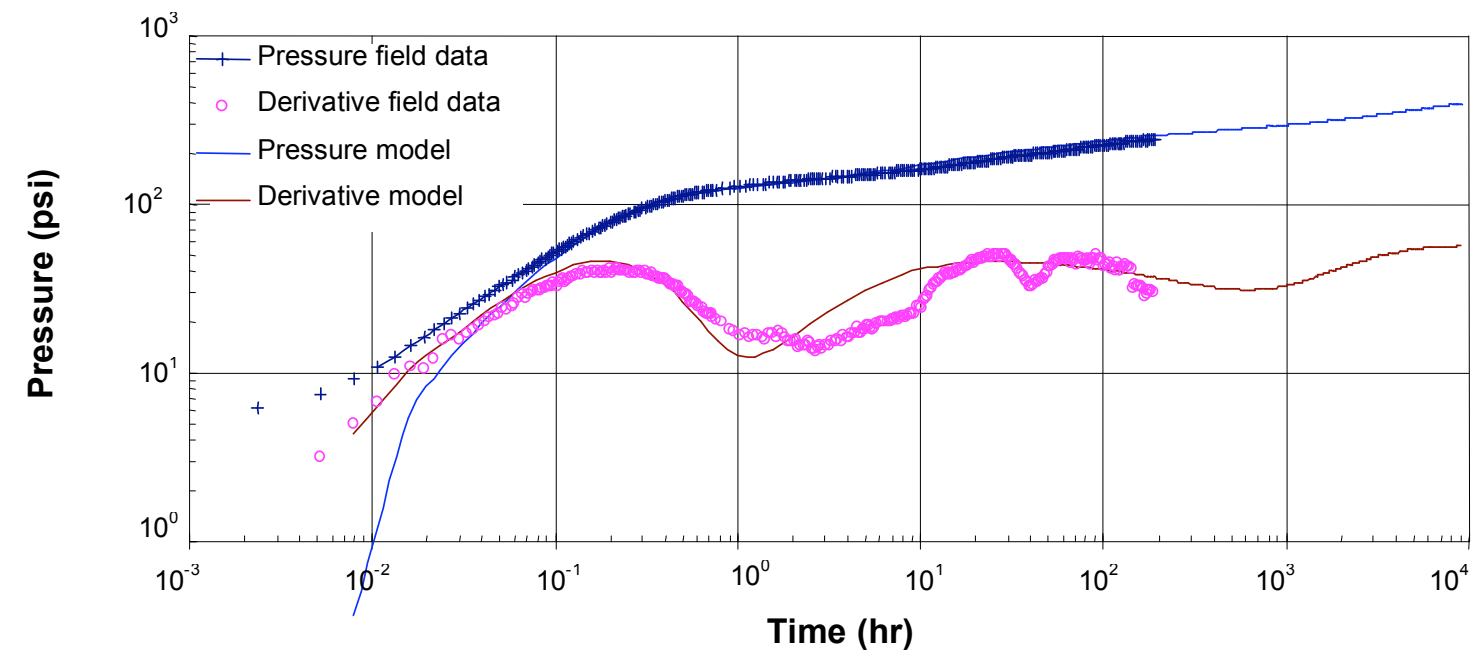

Figure 5. Comparison between measured pressure drawdown and its derivative data and modeled predictions for Well TK609

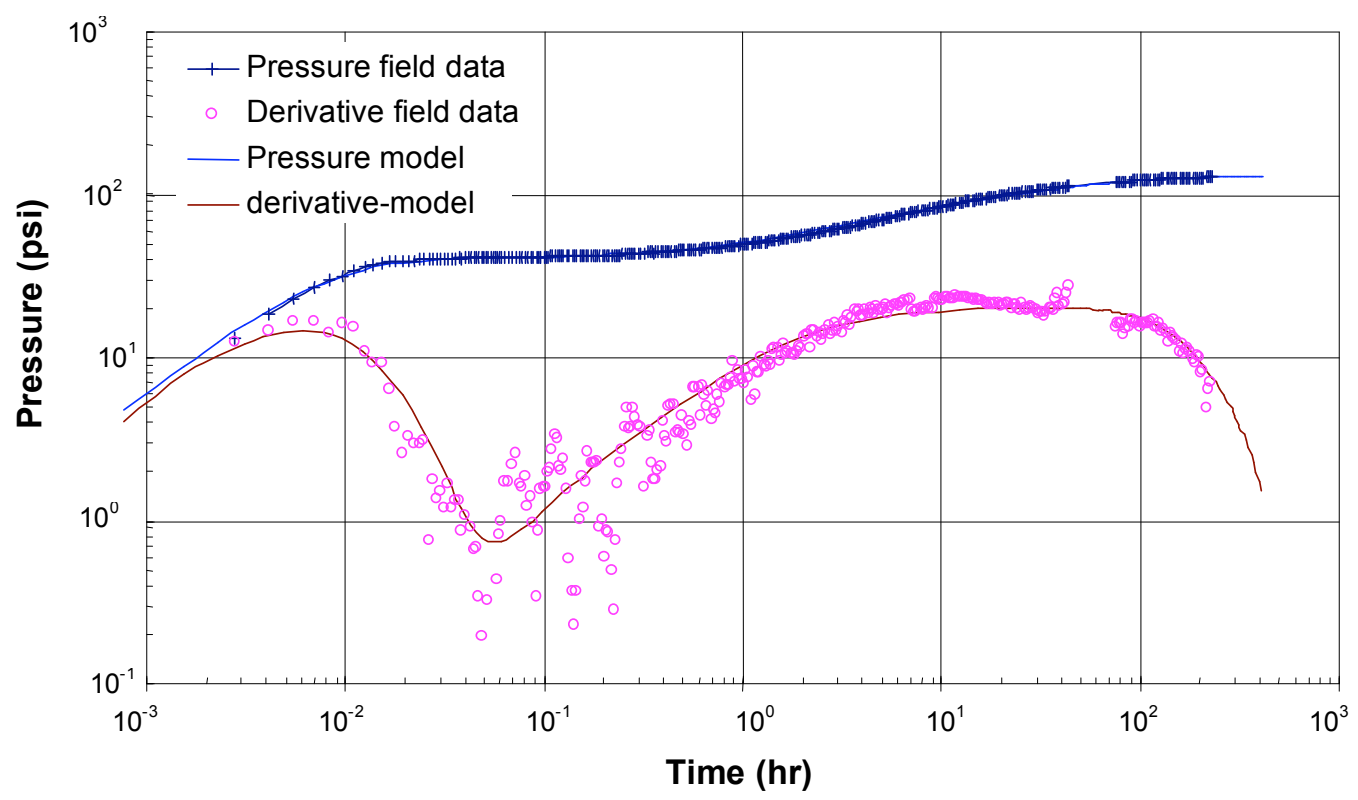

Figure 6. Alternate double-porosity pressure-transient analysis for field example 